\title{
«Ein Hospiz ist eine Herberge für Sterbende»
}

\section{Daniel Lüthi}

Freier Journalist und Fotograf, Medientrainer, Bern

Katze Mimi begrüsst und lässt sich dann nicht mehr blicken. Luise Thut zeigt die paar Rosentöpfe auf dem Balkon - «in unserem Garten hatte ich 150 Stöcke», kommentiert sie, und etwas Wehmut schwingt mit. Ein paar kleine Flaschen Prosecco stehen auch da. Sicher für den runden Geburtstag, ist der Besucher überzeugt. "Geburtstag?», erwidert die schicke und zierliche Dame mit den klaren blauen Augen - «dazu hatte ich noch nie ein besonderes Verhältnis. Jeder Tag ist ein geschenkter Tag.»

\section{Das Konzept Hospiz}

Dieses Jahr ist es unmöglich, auszuweichen: Viele um sie herum wollen den grossen runden Festtag mit ihr feiern, wollen sie feiern, geplant ist unter anderem ein Mittagessen mit einer Parlamentarierin im Bundeshaus. Sie selber hat Weggefährtinnen und Weggefährten zu einem Apéro riche eingeladen. Ansonsten bemüht sie sich einmal mehr, ihre Person hinter ihr Projekt zu stellen: «Es geht nicht um mich, es geht um

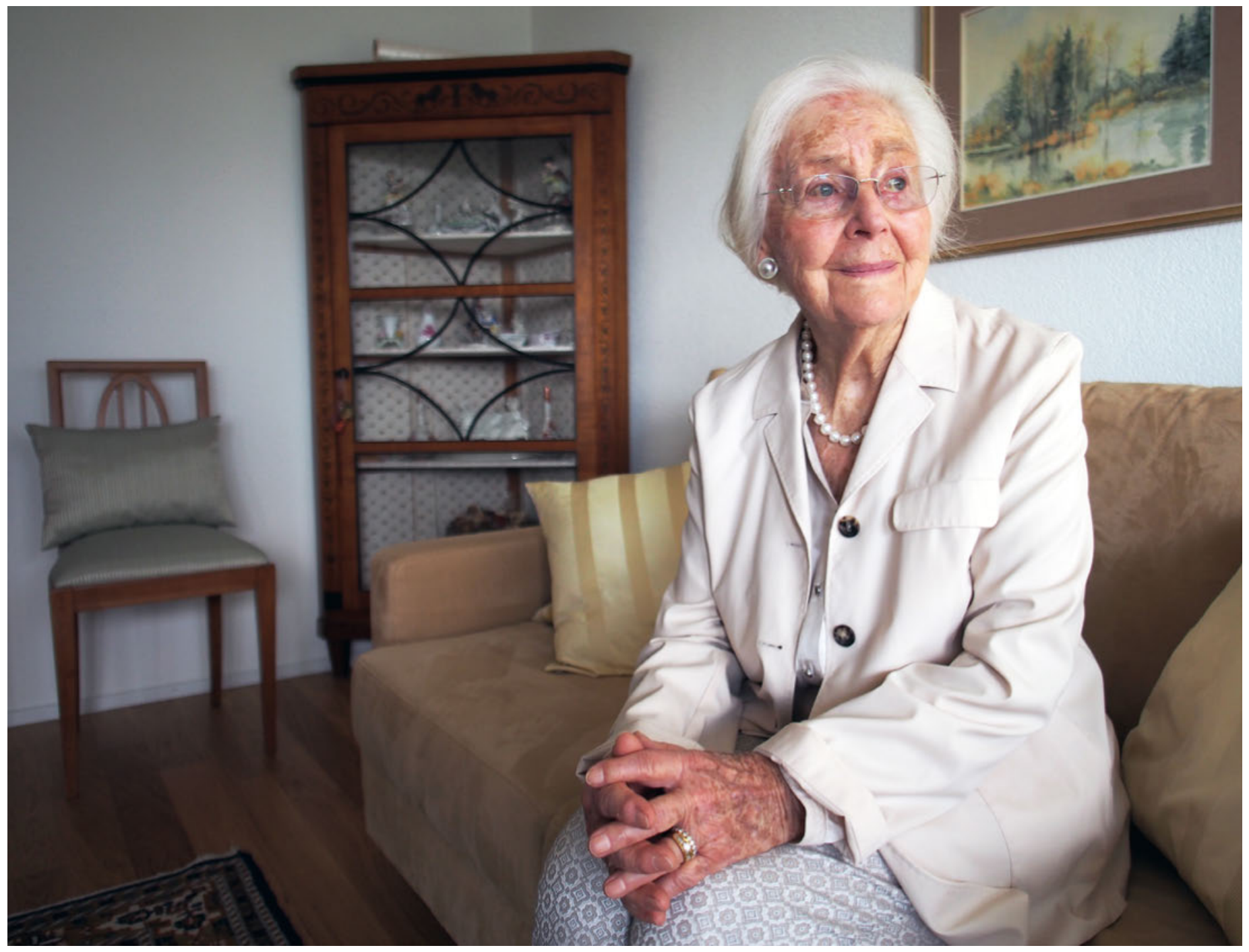


das Hospiz», sagt sie. Jeder habe eine Lebensaufgabe, erklärt sie-das Hospiz ist ihre.

Und wenn sie darüber spricht, spricht sie auch über Sarah. Denn mit, bei und dank ihr begann der lange und beschwerliche Kampf von Luise Thut für ihr Lebenswerk. Sarah war Thuts Freundin und eine der prägenden Personen ihres Lebens. Sie arbeitete als USKonsulin in Zürich, 1984 erhielt sie die Diagnose Brustkrebs. 1989 starb sie in den USA an ihrem Tumor. Luise Thut besuchte sie regelmässig und lernte dank ihr in der Nähe von Washington ihr erstes Hospiz kennen. "Als ich diesen Ort das erste Mal betrat, begegnete ich einer Frau, die Kleider bügelte und dabei laut sang", erinnert sie sich. Heiterkeit und Herzlichkeit in einem solchen Haus: Das beeindruckte sie.

Nach dem Tod ihrer Freundin beschloss Luise Thut «mit Sarah zusammen», wie sie betont -, das Konzept «Hospiz» in die Schweiz zu bringen und hier zu verankern. Mit viel Beharrlichkeit und gegen einige Widerstände tat sie dies dann auch. Weil sie realisierte, dass es hierzulande bei der Betreuung und Begleitung von Schwer- und Schwerstkranken eine Lücke gab: Die Infrastruktur eines Akutspitals war häufig zu teuer und nicht mehr nötig, und eine Betreuung durch die Angehörigen zu Hause nicht möglich.

\section{Ein Ort der Geborgenheit}

«Es geht um die Bedeutung eines Menschen, auch dann, wenn er krank ist», erklärt Luise Thut. «Es geht darum, einem Menschen, der sich nutzlos und überflüssig fühlt oder der Angst vor dem Sterben hat, rund um die Uhr einen Ort der Geborgenheit und Liebe zu bieten.» In einem Spital fehlten für eine 24-Stunden-Betreuung halt die Zeit und das Personal, im Übrigen stünden dort die medizinische Betreuung und damit das Heilen im Vordergrund. In einer Palliativstation sei der Aufenthalt eines Sterbenden durch die Vorgaben der Krankenversicherungen limitiert, auch hier gebe es also Unterschiede zu dem, was sie im Sinn gehabt habe. Ein Hospiz zeichne sich durch einen ganzheitlichen, lebensbejahenden Ansatz aus, ganz nach dem Motto von Cicely Saunders, einer anderen Schlüsselperson ihres Lebens: «Es geht darum, den Tagen mehr Leben, und nicht, dem Leben mehr Tage zu geben.» Ein Hospiz sei ein Ort, der den Weg zum Übergang ebne, sagt Luise Thut: «Es ist wichtig, in welchem Zustand wir den Übergang vom Leben in den Tod machen. Ein Hospiz ist eine Herberge für Sterbende - ein Zuhause, eine Heimat für die letzten Tage.»

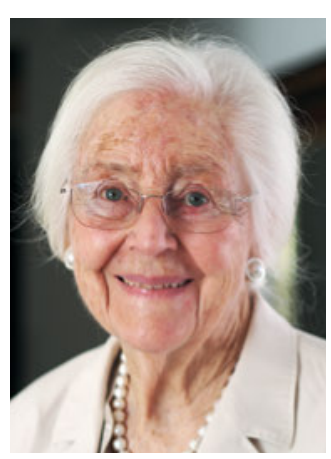

\section{Zur Person}

Luise Thut wurde am 28. Februar 1928 in München geboren. Sie ist also eben 90 Jahre alt geworden. Nach dem Krieg trennten sich ihre Eltern. 1952 kam sie mit einem Studentenvisum zu Verwandten nach New York, wo sie als Kindererzieherin arbeitete. 1954 reiste sie, weil es mit einem Studium in den USA nicht klappte, zurück nach Deutschland. Hier wollte sie jedoch nicht bleiben und ging zurück nach New York. Einige Jahre lang arbeitete sie für die skandinavische Fluggesellschaft SAS auf dem Flughafen JFK, dann lernte sie ihren späteren Mann Heinz Thut kennen. Mit ihm - einem Swissair-Piloten - kehrte sie 1965 nach Europa zurück. Im aargauischen Zufikon baute sich das Ehepaar ein Haus, das Luise Thut bis vor kurzem bewohnte.

In den 80er Jahren lernte sie dank ihrer Freundin Sarah in der Nähe von Washington ihr erstes Hospiz kennen. Nach dem Tod von Sarah beschlossThut, das Konzept «Hospiz» in die Schweiz zu bringen. Gestärkt durch die Begegnungen mit Cicely Saunders und Elisabeth Kübler-Ross sowie die Ausbildung zur Certified Hospice-Trainerin verwirklichte sie ihr Lebensprojekt auch.

1994 gründete sie den Aargauer Hospiz-Verein zur Begleitung Schwerkranker, 1998 die Luise-Thut-Stiftung. 2005 eröffnete sie das erste stationäre Hospiz im Kanton Aargau, wo Pflegefachpersonen und Freiwillige arbeiteten. 2010 übersiedelte dieses ins ehemalige Spital Brugg. 2008 trat Luise Thut als Präsidentin ihres Vereins zurück. Seit ein paar Wochen lebt sie mit ihrer Katze Mimi in einer altersgerechten Wohnung in Berikon.

\section{Die Alterswohnung}

Erst vor ein paar Wochen ist Luise Thut in ihr neues Zuhause in Berikon umgezogen. Die Dreieinhalbzimmerwohnung ist altersgerecht und hell, sie bietet auch einen schönen Ausblick nach Friedlisberg und weiter. Das ehemalige Haus, das rund 50 Jahre lang ihr $\mathrm{Zu}$ hause war, liegt nur ein paar hundert Meter entfernt und ist inzwischen verkauft worden. Am neuen Ort wollte Luise Thut eine Wohnung ganz oben, im dritten Stock, und die hat sie erhalten. Das habe eher mit Glück als mit Durchsetzungsvermögen zu tun, sagt sie, und macht eine kleine Führung.

Im Wohnbereich und im dritten Zimmer stehen Biedermeiermöbel aus der Kindheit, ein kleiner Tisch und eine Eckvitrine zum Beispiel. Eine Reihe japanische «Wood Block Prints» aus dem 19. Jahrhundert erinnern an Besuche in Tokyo, zwei Gobelinstühle an die handwerklichen Fähigkeiten der Grossmutter. «Das ist auch schon eine Weile her», scherzt Thut - tatsächlich: Auch diese Stühle sind Zeugen aus dem vorletzten Jahrhundert. Im Fernseher läuft wie immer 
klassische Musik. Wagner ist Thut besonders lieb, die negativen politischen Kommentare zu diesem Komponisten interessieren sie nicht, «Musik soll offen sein für alle», sagt sie.

\section{Der Mann des Lebens}

Dank ihrem Mann ist die Schweiz zu Luise Thuts Land geworden. Und ihr Mann ist die wichtigste Person in ihrem Leben, nach wie vor, deshalb war sein Tod 2013 für sie ein besonders schwerer Schlag. Er war an Krebs erkrankt und konnte nicht geheilt werden. «Ein grosser Schmerz», sagt Luise Thut. Und ergänzt: "Aber wir gingen beide immer auch unseren eigenen Weg. Wir haben uns gegenseitig immer gehen lassen. Und Heinz war Pilot, er ging immer wieder.»

Auch nach seinem Tod sei sie mit ihm geistig verbunden geblieben, sagt sie. «Ich lebe mit ihm und liebe ihn wie eh und je.» Mit seinem Tod habe sie einen Reifeprozess durchlebt, der sie schliesslich auch dazu befähigt habe, andere Trauernde zu trösten. «Das kann nur, wer selber einen grossen Verlust erlebt hat. Wobei es Trost eigentlich gar nicht gibt. Ich kann bloss dasitzen, die Hand reichen, zuhören - und zu etwas Erleichterung verhelfen.» Übrigens: Bis Luise Thut so weit war, dass sie in ihrem Haus ein Bild ihres verstorbenen Mannes aufhängen mochte, dauerte es ein Jahr.

Das gemeinsame Leben führte den Swissair-Captain und seine Frau immer wieder in die weite Welt, so verbrachten sie ein paar Jahre in Bangkok und in Hongkong. In Bangkok begegnete Luise Thut 1971 einer buddhistischen Nonne, die ihr neue religiöse und philosophische Dimensionen eröffnete. «Der Katholizismus hat mir nie eine seelische Heimat gegeben", sagt sie, «ich empfand ihn immer als angstmachend.» Im Buddhismus aber fand sie Grundsätze, nach denen sie sich richten wollte. Zum Beispiel: «Wir sind nicht nur geboren worden, um Spass zu haben, sondern vor allem, um etwas zu lernen. Und um etwas zu tun.» Oder: "Wie kannst du glücklich werden? Indem du einen anderen Menschen glücklich machst. So gesehen ist das Hospiz Aargau das schönste Geschenk in meinem Leben.»

\section{Umgang mit Sterben und Tod}

Die Hospiz-Bewegung brachte Thut auch mit Elisabeth Kübler-Ross in Kontakt. Mehrmals besuchte Thut die berühmte Psychiaterin und Pionierin beim Thema Sterbehilfe, auch kurz vor ihrem Tod 2004 in Scottsdale, Arizona. Das eigene Sterben zu akzeptieren, um anderen Sterbenden beistehen zu können, war einer ihrer Hauptgedanken. Und dass der Tod nicht ein Ende dere Existenz. Ihr eigener Tod sei für Kübler-Ross dann keine einfache Sache gewesen, erinnert sich Luise Thut. «Sie wehrte sich dagegen, indem sie sich beispielsweise als Sterbende noch einen Indoor-Swimmingpool bauen liess. Sie kokettierte mit dem Tod - und ja: Es ist möglich, dass sie Angst vor dem Sterben hatte.»

Interessant, welche Erfahrungen Luise Thut - bezogen auf ihr Thema - auf ihrem langen Weg mit Ärztinnen und Ärzten gemacht hat. "Cicely Saunders zum Beispiel war Krankenschwester. Die Ärzte boykottierten sie und nahmen sie erst ernst, nachdem sie Medizin studiert hatte.»

Thut hat nicht Medizin studiert. Sie eignete sich ihr Wissen im Laufe der Jahre in zahlreichen Kursen und Praktika an und mit einem Ausbildungsgang in Clearwater, Florida, den sie 1996 als «Certified Hospice Trainer» abschloss. Auch ihr und ihren Plänen gegenüber begegneten Ärzte immer wieder ablehnend. "Sie können sich gar nicht vorstellen, wie viel Widerstand mir von medizinischer Seite immer wieder entgegenkam", berichtet Thut, «aber die meisten Ärztinnen und Ärzte wollen mit dem Sterben ohnehin nicht konfrontiert werden. Im Übrigen ist die Medizin heute ja so weit fortgeschritten, dass wir bald Angst haben müssen, ob wir überhaupt noch sterben dürfen.»

\section{Und das eigene Ende?}

Etwas Humorvolles tönt aus dieser Antwort. Das ermutigt, die Frage zu stellen, die bei einer 90-Jährigen zwar naheliegend, gleichzeitig aber mit Hemmungen verbunden ist: Und Sie selber? Wie halten Sie es mit dem Sterben und dem Tod? «Ich bin grundsätzlich kein ängstlicher Mensch», antwortet Luise Thut ganz selbstverständlich. "Ich werde ohne Wehmut gehen - und mit dem Vertrauen, dass es schon richtig kommt. Auch weiss ich, dass jeder Mensch abgeholt wird. Keiner geht alleine rüber.»

Auf einer kleinen Fotografie an der Wand neben dem Tisch ist ihre Mutter zu sehen. «Sie starb mit 103 Jahren. Kurz vor ihrem Tod sagte sie mir: 'Du kannst dir gar nicht vorstellen, wer alles schon da ist.'»

Zum Schluss wartet Luise Thut noch mit einer Überraschung auf. Für sich selber hat sie für die letzten Tage bereits vorgesorgt, das war zu erwarten. «Ich möchte, wenn immer möglich, hier in meiner Wohnung sterben", sagt sie. Jetzt aber steht sie auf und zeigt eine Einzimmerwohnung gleich nebenan, die sie dazugemietet hat. Zurzeit nutzen sie Besucherinnen und Besucher. Später einmal wird sie für Betreuende und Pflegende reserviert sein. Zusammen also sind die beiden Wohnungen das private kleine Hospiz der HospizPionierin Luise Thut. 